\title{
Acral Verrucous Microcystic Lymphangioma Circumscriptum of the Toes Accompanying Local Hypertrichosis
}

The Editor,

Sir,

According to the 1996 classification of the International Society for the Study of Vascular Anomalies (ISSVA)/Mulliken, vascular anomalies may be classified as simple (capillary, lymphatic, venous and arterial) or combined (1). Lymphangiomas account for $4 \%$ of all vascular malformations and $26 \%$ of all benign vascular tumours (2). It is either primary (usually present at birth) or secondary [as a result of an acquired damage] $(3,4)$. When viewed from another aspect, the localized lymphatic malformations may be macrocystic or microcystic. The macrocystic form is known as cystic hygroma which is a large and soft-lesion covered with flesh or bluish-coloured skin and usually located on the back of the neck.

The microcystic forms is also known as Lc (1). It is characterized by clustered (localized) or diffused (classic), translucent vesicles 1 to $5 \mathrm{~mm}$ in diameter and filled with clear lymphatic fluid (3). Congenital or primary forms represent the malformation of deep dermal and subcutaneous lymphatics with a secondary dilatation of superficial lymphatics whereas acquired forms (or lymphangiectasia) represent dilatation of superficial lymphatics, resulting from the obstruction of previously normal deep lymphatics (5). The most common form is the vesicular form (1). However, it may also present in the form of verrucous lesions $(3,5)$. Presentation as a verrucous plaque limited to only toes is unusual (1).

We report a 50-year old Caucasion female with verrucous papules on the second and third toes of her right-foot that was progressively increasing in size and associated with pain and itching when walking. According to the history, a drawer dropped on her right-foot one and half years ago. Over the time, the second and third toes of the right-foot gradually began to swell. Eight months ago, multiple small protrusions occured on the swellings and four months ago black-coloured and thin hair growth began on the second toe. The patient visited five general practitioners previously and they stated that the lesions were warts. A 5-FU solution prescribed did not cure the lesions.

Dermatological examination revealed significant nonpitting and hard oedema and multiple, flesh-colored warty papules which were $2-5 \mathrm{~mm}$ in size on the second and third toes of the oedematous right-foot and localized hypertichosis on the toes (Fig. 1). No whitening of the lesions was observed with the application of 5\% acetic acid solution. There was no palpable inguinal lymph nodules were also absent. There was no additional pathology detected on further dermatological and physical examination (including genito-urinary system), routine laboratory tests, abdominal and pelvic ultrasonography

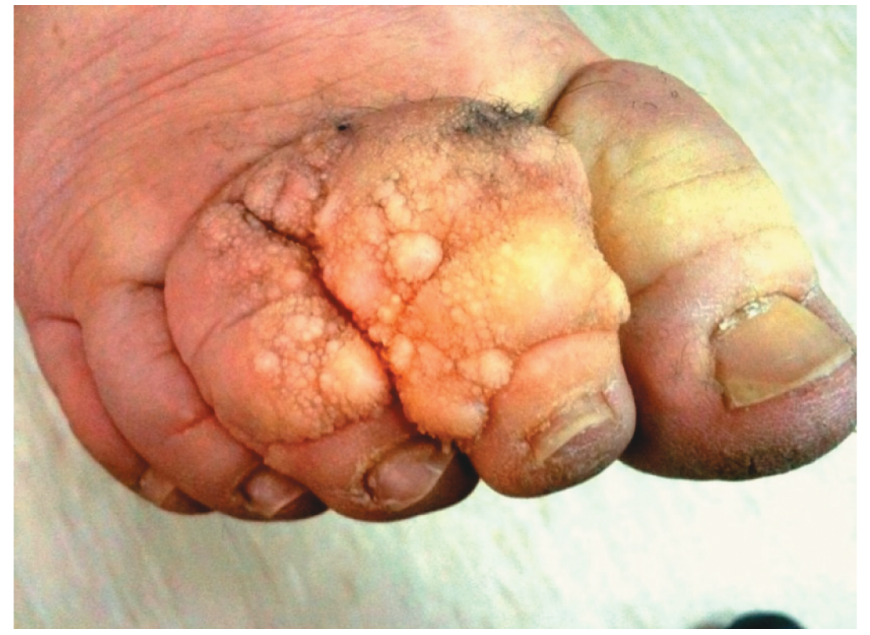

Fig. 1: Multiple small verrucous tumours on the second and third toes, and local hypertrichosis on the second toe of the oedematous right-foot.

and bilateral Doppler angiography of the legs. Purified protein derivative (PPD) skin test was negative. An excisional biopsy specimen was obtained from the lesions of the third toe.

Histopathological examination revealed an epidermal moderate achantosis, a prominent verruciform hyperkeratosis and numerous dilated, tortuous, microcystic lymphatic proliferations in the papillary and reticular dermis. There was no koilocytosis and the endothelial cells of the lymphatics did not have any atypia (Fig. 2a,b). The immunohistochemical examination revealed a marked luminal staining with D2-40 in the

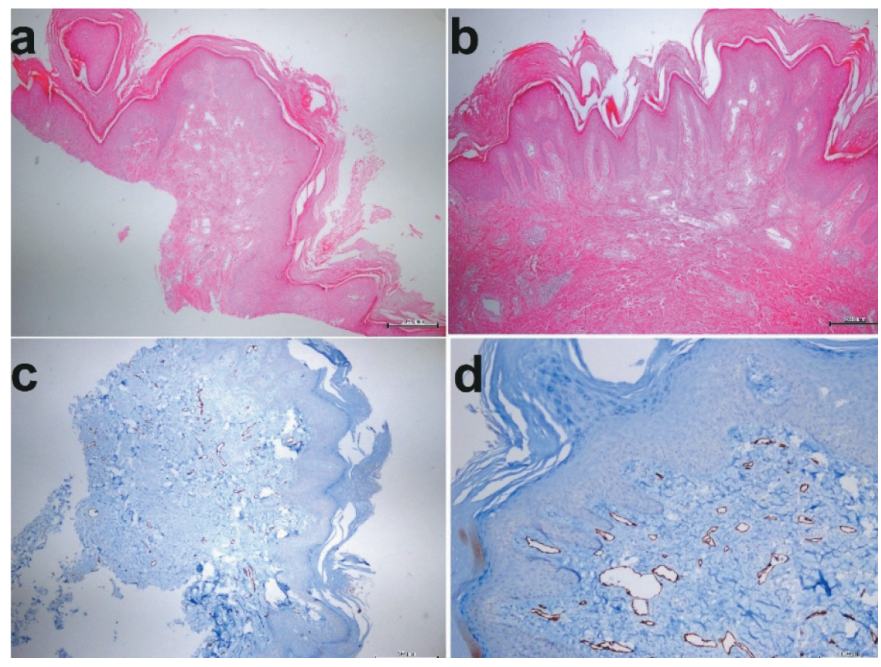

Fig. 2 (a, b): Epidermal achantosis, prominent verruciform hyperkeratosis and microcystic lymphatic proliferations in papillary and reticular dermis ( a) H\&EX40, b) H\&E X 100).

(c, d): Positive luminal staining of the lymphatic endothelial cells with D2-40 immunohistochemistry ( c) D2-40 X 40, d) D2-40 X 200). 
dermal lymmphatics (Fig. 2c, d). The findings led to the diagnosis of acral verrucous microcystic lymphangioma. After the diagnosis a pulsed-dye laser treatment was planned.

The localized forms of lymphangiomas occur less frequently and appear as small, discrete vesicular lesions that have no predilection for any site or any age. The axilla, adjacent chest-wall, oral cavity and tongue are common sites (3, 4). Acquired lymphangiomas are rare and have appeared after radiotherapy to carcinoma of cervix, tuberculous inguinal adenitis, recurrent cellulitis, intra-abdominal and pelvic surgeries, scrofuloderma, scarring after keloids and Crohn's disease (4, 5). Although the aetiology of the acquired Lc is uncertain, it has been stated that the lesions occured after the trauma. However, only a few cases of late onset developed in adulthood is reported in literature which began after a trauma (1) like the case reported in this study. The verrucous forms have been more frequently reported in the vulvar region $(3,5)$. Rarely, local hypertrichosis may occur .

The first case of acral verrucous Lc whose lesion was limited to the toe has been reported by Juca et al in 2011. Their patient had not any trauma history. But the authors stated that the situation should be considered with caution, since toes are often subject to micro-trauma (1). To the best of our knowledge, this is the second acral verrucous Lc case and the first example where it is accompanied by local hypertrichosis in the literature. The main histopathological finding of a $\mathrm{Lc}$ is the dilated lymphatics in the papillary dermis (5). Some of these lymphatics may have small intraluminal projections with a papillary appearance beneath the epidermis. Hyperkeratosis and acanthosis may be present in variable degrees (1). It has been proposed that the verrucous appearance might have been due to the hyperkeratosis (3). Scattered lymphocytes in the stroma may be seen (5).

The presence of blood inside the vessels may be supportive of a recent haemorrhage or a combined malformation, generally venous and lymphatic (1). The lymphatic endothelial cells have been found to be CD31 and D2-40 positive (5). We also obtained similar findings in the histopathology as described in the literature. Diagnosis is primarily based on histo- pathological findings. In the differential diagnosis, angioma, angiokeratoma, glomangioma, molluscum contagiosum (1), viral warts (5) and tuberculosis cutis verrucosa should be considered (3). Treatment options include surgical excision, cryosurgery, electrocautery, surface ablation with laser, sclerotherapy with sclerosing agents like OK-432, radiotherapy $(3,5)$ and lymphatico-venular anastomosis (4). In conclusion, this case is presented with the aim to draw attention to these very rare and unique lesions and when with such warty lesions on the acral locations to avoid the wrong treatments and redundant interventions. An acral verrucous microcystic Lc should be considered in the differential diagnosis.

Keywords: Acquired, acral, lymphangioma circumscriptum, wart

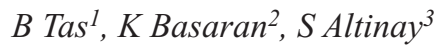

From: ${ }^{1}$ Department of Dermatology, ${ }^{2}$ Department of Aesthetic, Plastic and Reconstructive Surgery and ${ }^{3}$ Department of Pathology, Health Sciences University, Bagcilar Research and Training Hospital Istanbul/Turkey.

Correspondence: B Tas, Atakoy 7-8. Klsım, Martı Sitesi, 14/105, Bakırkoy/Istanbul, 34156. Fax: (90) 212-4404000. e-mail:betulavc@yahoo.com.

\section{REFERENCES}

1. Juca NB, Crisostomo MG, Oliveira LM, Cavalcante HA, Sousa AR. Acral microcystic lymphangioma: differential diagnosis in verrucous lesions of extremities. An Bras Dermatol 2011; 86: 343-6.

2. Mehta V, Nayak S, Balachandran C, Monga P, Rao R. Extensive congenital vulvar lymphangioma mimicking genital warts. Indian J Dermatol 2010; 55: 121-2.

3. Aggarwal K, Gupta S, Jain VK, Marwah N. Congenital lymphangioma circumscriptum of the vulva. Indian Pediatr 2009; 46: 428-9.

4. M Gönül, SK Çakmak, S Soylu, A Kılıç, H Gündüz, Ü Gül, Ünal T. Lymphangioma circumscriptum of the vulva: a case report. Acta Derm Venereol 2009; 89: 180-1.

5. Gnanaraj P, Revathy V, Venugopal V, Tamilchelvan D, Rajagopalan V. Secondary lymphangioma of vulva: a report of two cases. Indian J Dermatol 2012; 57: 149-51. 